\title{
Article
}

\section{Invariant Quantum States of Quadratic Hamiltonians}

Viktor V. Dodonov ${ }^{1,2}$ (D)

check for updates

Citation: Dodonov, V.V. Invariant Quantum States of Quadratic

Hamiltonians. Entropy 2021, 23, 634 https://doi.org/10.3390/e23050634

Academic Editor: Alessandro Sergi

Received: 30 April 2021

Accepted: 16 May 2021

Published: 19 May 2021

Publisher's Note: MDPI stays neutral with regard to jurisdictional claims in published maps and institutional affiliations.

Copyright: (c) 2021 by the authors. Licensee MDPI, Basel, Switzerland. This article is an open access article distributed under the terms and conditions of the Creative Commons Attribution (CC BY) license (https:// creativecommons.org/licenses/by/ $4.0 /)$.
1 Institute of Physics, University of Brasilia, P.O. Box 04455, Brasilia 70919-970, DF, Brazil; vdodonov@unb.br

2 International Center for Physics, University of Brasilia, Brasilia 70919-970, DF, Brazil

Abstract: The problem of finding covariance matrices that remain constant in time for arbitrary multi-dimensional quadratic Hamiltonians (including those with time-dependent coefficients) is considered. General solutions are obtained.

Keywords: covariance matrix; positively (semi)definite matrices; symplectic transformations; charged particle in homogeneous magnetic fields; generalized frequency converter

\section{Introduction}

I consider a closed quantum system described by means of a homogeneous quadratic Hamiltonian

$$
H=\frac{1}{2} \sum_{j, k=1}^{2 N} B_{j k}(t) q_{j} q_{k}=\frac{1}{2} \mathbf{q} B(t) \mathbf{q} .
$$

Here, $\mathbf{q}$ is the $2 N$-dimensional vector, whereas $B$ is a $2 N \times 2 N$ matrix. This vector and matrix are divided into the $N$-dimensional blocks as follows,

$$
\mathbf{q}=\left[\begin{array}{l}
\mathbf{p} \\
\mathbf{x}
\end{array}\right], \quad B=\left\|\begin{array}{ll}
b_{1} & b_{2} \\
b_{3} & b_{4}
\end{array}\right\| .
$$

It is assumed that $\mathbf{x}$ and $\mathbf{p}$ are the $N$-dimensional vectors of the Cartesian coordinates and conjugated momenta, with $N$ being the number of degrees of freedom of the system. The $N \times N$ matrices $b_{j}$ may be arbitrary functions of time $(j=1,2,3,4)$. Evidently, matrix $B$ can always be symmetrized; thus, I assume that $b_{1}=\tilde{b}_{1}, b_{4}=\tilde{b}_{4}, b_{2}=\tilde{b}_{3}$; the tilde means the matrix transposition. If the Hamiltonian is Hermitian, then matrix $B$ is real.

Many statistical properties of the quantum system are contained in the $2 \mathrm{~N} \times 2 \mathrm{~N}$ symmetric covariance matrix

$$
\mathcal{M}=\left\|\overline{q_{j} q_{k}}\right\|=\left\|\begin{array}{ll}
\mathcal{M}_{p p} & \mathcal{M}_{p x} \\
\mathcal{M}_{x p} & \mathcal{M}_{x x}
\end{array}\right\|, \quad \mathcal{M}_{p x}=\tilde{\mathcal{M}}_{x p}
$$

where the (co)variances are defined as

$$
\overline{q_{j} q_{k}}=\frac{1}{2}\left\langle\hat{q}_{j} \hat{q}_{k}+\hat{q}_{k} \hat{q}_{j}\right\rangle-\left\langle\hat{q}_{j}\right\rangle\left\langle\hat{q}_{k}\right\rangle
$$

and the splitting of matrix $\mathcal{M}$ in $N \times N$ blocks is performed in accordance with the structure of the $2 N$-dimensional vector $\mathbf{q}=(\mathbf{p}, \mathbf{x})$. Formula (4) can be re-written as

$$
\frac{1}{2}\left\langle\hat{q}_{j} \hat{q}_{k}+\hat{q}_{k} \hat{q}_{j}\right\rangle=C_{j k}+Q_{j k}, \quad C_{j k}=\left\langle\hat{q}_{j}\right\rangle\left\langle\hat{q}_{k}\right\rangle, \quad Q_{j k}=\overline{q_{j} q_{k}} .
$$

The remarkable property of quadratic Hamiltonians is the total independence of the first-order mean values $\left\langle\hat{q}_{j}\right\rangle$ and the covariances $\overline{q_{j} q_{k}}$. Moreover, the evolution of the mean values $\left\langle\hat{q}_{j}\right\rangle$ is governed by the classical equations of motion. Therefore, Formula (5) demonstrates that the average value of any symmetric bilinear combination of canonical 
coordinate and momentum operators is the sum of two independent parts: the classical part $C_{j k}$ and the quantum part $Q_{j k}$, which is determined by quantum fluctuations only.

If one is concerned with the quantum properties of the system under study, then, the main object is the covariance matrix $\mathcal{M}$. Its evolution is governed by the equation

$$
d \mathcal{M} / d t=\mathcal{M} B(t) \Sigma-\Sigma B(t) \mathcal{M}, \quad \Sigma=\left\|\begin{array}{cc}
0 & E_{N} \\
-E_{N} & 0
\end{array}\right\|,
$$

where $E_{N}$ is the $N \times N$ unity matrix. Therefore, matrix $\mathcal{M}$ depends on time in the most general case.

Recently, the authors of [1] introduced the concept of invariant states, i.e., quantum states with time-independent covariance matrices. They demonstrated the existence of such states in the case of $N=2$, considering an example of a frequency converter. The invariant covariance matrix must satisfy the special case of Equation (6):

$$
\mathcal{M B}(t) \Sigma=\Sigma B(t) \mathcal{M}
$$

To solve Equation (7) for the $4 \times 4$ matrix $\mathcal{M}$, the authors of [1] transformed the matrix equation into an equivalent set of 10 equations for the components of a vector constructed from 10 independent elements of this symmetric matrix.

The aim of the present study is to solve Equation (7) directly in the matrix form and analyze possible forms of the invariant covariance matrices, both for the constant matrices $B$ and for some specific time-dependent matrices $B(t)$.

\section{Simple Solutions for Time-Independent Hamiltonians}

If matrix $B$ does not depend on time, any time-independent solution to Equation (7) is the solution to Equation (6) as well. The simplest solution is $\mathcal{M}_{*}=\eta B^{-1}$ with $\eta>0$. If matrix $B$ is positively definite (as happens for physical Hamiltonians), then matrix $\mathcal{M}_{*}$ is positively definite automatically. However, physical covariance matrices must be not only positively definite, but they must satisfy a more strong restriction of positive definiteness of matrix $\mathcal{Y}=\mathcal{M}+i \hbar \Sigma / 2$ [2,3]. In particular, the following inequality must be satisfied:

$$
\operatorname{det} \mathcal{M} \geq(\hbar / 2)^{2 N} \text {. }
$$

Therefore, the coefficient $\eta$ must satisfy the inequalty $\eta \geq(\operatorname{det} B)^{1 / 2 N} \hbar / 2$. For example, considering the one-dimensional harmonic oscillator with the diagonal matrix $B=\operatorname{diag}\left(m^{-1}, m \omega^{2}\right)$, we have $\operatorname{det} B=\omega^{2}$, so that the minimal choice $\eta_{\min }=\hbar \omega / 2$ yields the covariance matrix of the vacuum state. The value $\eta_{n}=(2 n+1) \eta_{\min }$ corresponds to the covariance matrix of the $n$-th energy eigenstate.

One can easily check that any matrix $(\Sigma B)^{k} \Sigma$ (with integer $k$ ) also satisfies Equation (7). Due to the linearity of this equation, one can write a more general solution as

$$
\mathcal{M}=\sum_{k= \pm 1, \pm 3, \ldots} c_{k}(\Sigma B)^{k} \Sigma,
$$

where the coefficients $c_{k}$ must be chosen in such a way that matrix $\mathcal{Y}$ is positively definite. The absence of even powers of matrix $\Sigma B$ in the sum (series) (9) is explained by the fact that all matrices $(\Sigma B)^{2 k} \Sigma$ are antisymmetric.

It is known that the covariance matrix does not depend on time for any quantum mixture of energy eigenstates, in particular, for the equilibrium states. For such states, we have $[3,4]$

$$
\mathcal{M}_{e q}=(\hbar / 2) \cot (\hbar \beta \Sigma \mathcal{B} / 2) \Sigma,
$$

where $\beta$ is the inverse temperature parameter. Formula (10) is the consequence of the general formula for the Gaussian Wigner function of homogeneous quadratic systems [3,5-7] 


$$
W_{\text {Gauss }}(\mathbf{q}) \sim \exp \left(-\frac{1}{2} \mathbf{q} \mathcal{M}^{-1} \mathbf{q}\right)
$$

and the explicit expression for the equilibrium Wigner function of the most general homogeneous quadratic Hamiltonians [8,9]

$$
W_{e q}(\mathbf{q}) \sim \exp \left[\hbar^{-1} \mathbf{q} \Sigma \tan (\hbar \beta \Sigma \mathcal{B} / 2) \mathbf{q}\right] .
$$

Formula (10) has the form of (9) with $k \geq-1$.

Matrix $(\Sigma B)^{2}$ has the following block form:

$$
(\Sigma B)^{2}=\left\|\begin{array}{cc}
\tilde{b}_{2}^{2}-b_{4} b_{1} & \tilde{b}_{2} b_{4}-b_{4} b_{2} \\
b_{2} b_{1}-b_{1} \tilde{b}_{2} & b_{2}^{2}-b_{1} b_{4}
\end{array}\right\|
$$

This matrix is proportional to the unit matrix if $N=1$, when all blocks are usual numbers. Consequently, the sum (series) (9) is proportional to $B^{-1}$ for all one-dimensional quadratic systems with non-degenerate matrix $B$. This means that one-dimensional systems possess a unique set of invariant covariance matrices, with the form $\eta B^{-1}$ (or an equivalent form $\xi \Sigma B \Sigma$ with $\xi<0$ in order to maintain the positive definiteness). This result can be easily checked by directly solving Equation (7) when all matrices have the dimension $2 \times 2$. In particular, any Gaussian invariant state can be considered as a thermal state with some effective temperature in the case of $N=1$.

Two-Dimensional Examples: A Charged Oscillator and a Charge in a Homogeneous Magnetic Field

However, the situation can be different for multidimensional systems. As an example of a two-dimensional system, let us consider an isotropic charged two-dimensional oscillator in the plane $x y$, placed in a homogeneous magnetic field $\mathcal{H}$ perpendicular to this plane. For the circular gauge of the vector potential, $\mathbf{A}=\mathcal{H}(-y, x, 0) / 2$, the Hamiltonian has the form (using dimensionless variables)

$$
\hat{H}=\frac{1}{2}\left(\hat{p}_{x}+\omega \hat{y}\right)^{2}+\frac{1}{2}\left(\hat{p}_{y}-\omega \hat{x}\right)^{2}+\frac{1}{2} g\left(\hat{x}^{2}+\hat{y}^{2}\right),
$$

so that the $2 \times 2$ blocks of $4 \times 4$ matrix $B$ can be written in the form

$$
b_{1}=E_{2}, \quad b_{2}=-\tilde{b}_{2}=\omega \Sigma_{2}, \quad b_{4}=-b_{2}^{2}+g^{2} E_{2}=\left(\omega^{2}+g^{2}\right) E_{2},
$$

where $\omega$ is the Larmor frequency and $g$ is the oscillator frequency. Using the Frobenius formula for the inversion of block matrices [10],

$$
\left\|\begin{array}{ll}
a & b \\
c & d
\end{array}\right\|^{-1}=\left\|\begin{array}{cc}
a^{-1}+a^{-1} b h^{-1} c a^{-1} & -a^{-1} b h^{-1} \\
-h^{-1} c a^{-1} & h^{-1}
\end{array}\right\|, \quad h=d-c a^{-1} b,
$$

one can obtain the following independent solutions to Equation (7) (containing the matrix $b_{2}$ with different signs):

$$
\mathcal{M}_{1}=g^{2} B^{-1}=\left\|\begin{array}{cc}
\left(\omega^{2}+g^{2}\right) E_{2} & -\omega \Sigma_{2} \\
\omega \Sigma_{2} & E_{2}
\end{array}\right\|, \quad \mathcal{M}_{2}=-\Sigma B \Sigma=\left\|\begin{array}{cc}
\left(\omega^{2}+g^{2}\right) E_{2} & \omega \Sigma_{2} \\
-\omega \Sigma_{2} & E_{2}
\end{array}\right\| \text {. }
$$

These matrices should be multiplied by some factors in order to obtain positively definite matrices satisfying the condition $\operatorname{det} \mathcal{M} \geq 1 / 16$, according to Equation (8). The determinants of the matrices $\mathcal{M}_{1,2}$ can be calculated with the aid of the Frobenius determinant formulas

$$
\operatorname{det}\left\|\begin{array}{ll}
a & b \\
c & d
\end{array}\right\|=\operatorname{det}(a) \operatorname{det}\left(d-c a^{-1} b\right)=\operatorname{det}(d) \operatorname{det}\left(a-b d^{-1} c\right) .
$$


Therefore, $\operatorname{det}\left(\mathcal{M}_{1}\right)=\operatorname{det}\left(\mathcal{M}_{2}\right)=\operatorname{det}(B)=g^{4}$. Consequently, matrices (14) cannot be used directly in the limit case of $g=0$ (a free charged particle in a homogeneous magnetic field). However, in this case, one can use linear combinations of $\mathcal{M}_{1}$ and $\mathcal{M}_{2}$ in the form (assuming $\omega>0$ )

$$
\mathcal{M}_{\gamma}=\frac{N}{2 \sqrt{1-\gamma^{2}}}\left\|\begin{array}{cc}
\omega E_{2} & \gamma \Sigma_{2} \\
-\gamma \Sigma_{2} & \omega^{-1} E_{2}
\end{array}\right\|, \quad|\gamma|<1, \quad \operatorname{det}\left(\mathcal{M}_{\gamma}\right)=N^{4} / 16 .
$$

The condition $\operatorname{det}\left(\mathcal{M}_{\gamma}\right) \geq 1 / 16$ is satisfied if $N \geq 1$. However, this is only a necessary condition of positivity of matrix $\mathcal{Y}=\mathcal{M}+i \hbar \Sigma / 2$. One has to also check the positivity of all principal minors of matrix $\mathcal{Y}$. However, in the case under study, one can use another way that sheds more light on the physical aspects of the problem.

It is known (at least since the paper [11]) that the following set of linear combinations of the canonical coordinates and momenta is very useful for the description of the motion of a free charged particle (of unit mass and charge) in the homogeneous magnetic field:

$$
\begin{aligned}
\hat{x}_{c} & =\frac{1}{2}\left(\hat{x}+\hat{p}_{y} / \omega\right), \quad \hat{y}_{c}=\frac{1}{2}\left(\hat{y}-\hat{p}_{x} / \omega\right), \\
\hat{x}_{r}=\hat{x}-\hat{x}_{c} & =\frac{1}{2}\left(\hat{x}-\hat{p}_{y} / \omega\right), \quad \hat{y}_{r}=\hat{y}-\hat{y}_{c}=\frac{1}{2}\left(\hat{y}+\hat{p}_{x} / \omega\right) .
\end{aligned}
$$

The first pair describes coordinates of the center of a circle where the particle rotates around, whereas the second pair of observables consists of two relative coordinates with respect to this center. The vectors $\mathbf{q}$ and $\mathbf{z}=\left(x_{r}, y_{r}, x_{c}, y_{c}\right)$ are related by means of the linear transformation $\mathbf{z}=U \mathbf{q}$ with

$$
U=\frac{1}{2}\left\|\begin{array}{cc}
-\omega^{-1} \Sigma_{2} & E_{2} \\
\omega^{-1} \Sigma_{2} & E_{2}
\end{array}\right\|, \quad U^{-1}=\left\|\begin{array}{cc}
\omega \Sigma_{2} & -\omega \Sigma_{2} \\
E_{2} & E_{2}
\end{array}\right\| .
$$

Hence, the covariance matrix $\mathcal{Z}$ with respect to the components of vector $\mathbf{z}$ has the form

$$
\mathcal{Z}_{\gamma}=U \mathcal{M}_{\gamma} \tilde{U}=\frac{N}{4 \omega}\left\|\begin{array}{cc}
E_{2} \sqrt{\frac{1+\gamma}{1-\gamma}} & 0 \\
0 & E_{2} \sqrt{\frac{1-\gamma}{1+\gamma}}
\end{array}\right\| .
$$

Due to the commutation relations

$$
\left[\hat{x}_{r}, \hat{y}_{r}\right]=\left[\hat{y}_{c}, \hat{x}_{c}\right]=i /(2 \omega),
$$

we have the uncertainty inequalities

$$
\sigma_{x_{r}} \sigma_{y_{r}} \geq(4 \omega)^{-2}, \quad \sigma_{x_{c}} \sigma_{y_{c}} \geq(4 \omega)^{-2} .
$$

Consequently, comparing Equations (18) and (20), one arrives at a more strong restriction

$$
N^{2} \geq(1+|\gamma|) /(1-|\gamma|)
$$

It is known that the covariance matrix determines the quantum state uniquely (up to an insignificant phase) if the state is Gaussian. In this case, the state is pure provided $\operatorname{det} \mathcal{M}=1$. Therefore, the only pure invariant Gaussian state of a charged particle in a homogeneous magnetic field has the covariance matrix (15) or (18) with $\gamma=0$. This is nothing but the coherent state introduced by Malkin and Man'ko in 1968 [12].

Now, let us note that the Hamiltonian (13) with $g=0$ can be rewritten in therms of the relative coordinates only:

$$
\hat{H}=2 \omega^{2}\left(\hat{x}_{r}^{2}+\hat{y}_{r}^{2}\right) .
$$


Since the pairs $\left(\hat{x}_{r}, \hat{y}_{r}\right)$ and $\left(\hat{x}_{c}, \hat{y}_{c}\right)$ are totally independent, the most general invariant $4 \times 4$ covariance matrix for the Hamiltonian (21) has the form

$$
\mathcal{Z}_{\text {gen }}=\left\|\begin{array}{cc}
\eta E_{2} & 0 \\
0 & \mathcal{C}
\end{array}\right\|, \quad \eta \geq(4 \omega)^{-1}, \quad \mathcal{C}=\left\|\begin{array}{cc}
c_{1} & c_{12} \\
c_{12} & c_{2}
\end{array}\right\|, \quad c_{1,2}>0, \quad c_{1} c_{2}-c_{12}^{2} \geq(4 \omega)^{-2} .
$$
matrix:

Returning to the $\mathbf{q}$-variables, one can obtain the following most general invariant

$$
\begin{gathered}
\mathcal{M}_{\text {gen }}=U^{-1} \mathcal{Z}_{\text {gen }} \tilde{U}^{-1}=\left\|\begin{array}{cc}
\omega^{2} G & \omega K \\
\omega \tilde{K} & F
\end{array}\right\|, \\
G=\left\|\begin{array}{cc}
\eta+c_{2} & -c_{12} \\
-c_{12} & \eta+c_{1}
\end{array}\right\|, \quad F=\left\|\begin{array}{cc}
\eta+c_{1} & c_{12} \\
c_{12} & \eta+c_{2}
\end{array}\right\|, \quad K=\left\|\begin{array}{cc}
-c_{12} & \eta-c_{2} \\
c_{1}-\eta & -c_{12}
\end{array}\right\| .
\end{gathered}
$$

Therefore, the most general invariant matrix is determined by three positive parameters $\left(\eta, c_{1}, c_{2}\right)$ and one parameter $\left(c_{12}\right)$ of an arbitrary sign. However, all these parameters must obey certain restrictions, given in Equation (22).

\section{General Solutions for Positive Time-Independent Hamiltonians}

The results of the preceding subsection show the general way to solve the problem in the case of an arbitrary positively definite time-independent matrix $B$. It is well known (since the paper [13], whose results can be found also in book [14]; see also [15-19]) that such matrices can be diagonalized by means of symplectic (canonical) transformations of the form

$$
T B \tilde{T}=B_{*}=\operatorname{diag}\left(B_{1}, B_{2}, \ldots, B_{N}\right), \quad T \Sigma \tilde{T}=\Sigma, \quad B_{j}=\operatorname{diag}\left(\mu_{j}, v_{j}\right), \quad \mu_{j}>0, \quad v_{j}>0 .
$$

Putting $B=T^{-1} B_{*} \tilde{T}^{-1}$ in Equation (7), one obtains an equivalent equation

$$
\mathcal{M}_{*} B_{*} \Sigma=\Sigma B_{*} \mathcal{M}_{*}, \quad \mathcal{M}_{*}=\tilde{T}^{-1} \mathcal{M} T^{-1} .
$$

Using the results of the preceding section related to the case of $N=1$, one can write the general solution:

$$
\mathcal{M}=\tilde{T} \operatorname{diag}\left(\eta_{1} B_{1}^{-1}, \eta_{2} B_{2}^{-1}, \ldots, \eta_{N} B_{N}^{-1}\right) T, \quad \eta_{j} \geq \hbar \sqrt{\mu_{j} v_{j}} / 2 .
$$

This depends on $N$ positive parameters $\eta_{j}$. However, the block matrices $B_{j}$ are not determined uniquely: only the products $\mu_{j} v_{j}$ are fixed by the eigenvalues of matrix $B$, while the ratio $\mu_{j} / \nu_{j}$ can be considered as an additional parameter. Therefore, the total number of parameters is $2 N$ for $N \geq 2$.

\section{Specific Time-Dependent Hamiltonians Admitting Invariant Covariance Matrices}

Equation (7) has an obvious solution $\mathcal{M}=\eta E_{2 N}$ for any matrix $B_{0}(t)$ satisfying the condition $B_{0} \Sigma=\Sigma B_{0}$. This results in the following relations between the $N \times N$ blocks:

$$
b_{1}=b_{4}, \quad \tilde{b}_{2}=-b_{2} .
$$

Multiplying both sides of the equality $B_{0} \Sigma=\Sigma B_{0}$ by some symplectic time-independent matrix $\Lambda$ from the left and matrix $\tilde{\Lambda}$ from the right, we find the following general set of solutions to Equation (7):

$$
\mathcal{M}=\eta \Lambda \tilde{\Lambda}, \quad B(t)=\tilde{\Lambda}^{-1} B_{0}(t) \Lambda^{-1} .
$$

Remember that symplectic matrices obey the relation

$$
\Lambda \Sigma \tilde{\Lambda}=\Sigma .
$$


The consequence is $|\operatorname{det} \Lambda|=1$. Hence, $\eta \geq \hbar / 2$. Clearly, matrix $\Lambda \tilde{\Lambda}$ is positively definite. Another consequence of the identity (28) is the formula $\Lambda^{-1}=-\Sigma \tilde{\Lambda} \Sigma$. Therefore,

$$
B(t)=\Sigma \Lambda \Sigma B_{0}(t) \Sigma \tilde{\Lambda} \Sigma .
$$

Finally, we obtain the following expressions for $2 N \times 2 N$ matrices in terms of $N \times N$ blocks:

$$
\begin{gathered}
\Lambda \tilde{\Lambda}=\left\|\begin{array}{cc}
\lambda_{1} & \lambda_{2} \\
\lambda_{3} & \lambda_{4}
\end{array}\right\| \cdot\left\|\begin{array}{cc}
\tilde{\lambda}_{1} & \tilde{\lambda}_{3} \\
\tilde{\lambda}_{2} & \tilde{\lambda}_{4}
\end{array}\right\|=\left\|\begin{array}{cc}
\lambda_{1} \tilde{\lambda}_{1}+\lambda_{2} \tilde{\lambda}_{2} & \lambda_{1} \tilde{\lambda}_{3}+\lambda_{2} \tilde{\lambda}_{4} \\
\lambda_{3} \tilde{\lambda}_{1}+\lambda_{4} \tilde{\lambda}_{2} & \lambda_{3} \tilde{\lambda}_{3}+\lambda_{4} \tilde{\lambda}_{4}
\end{array}\right\|, \\
B(t)=\left\|\begin{array}{cc}
\lambda_{4} \alpha \tilde{\lambda}_{4}+\lambda_{3} \alpha \tilde{\lambda}_{3}+\lambda_{3} \beta \tilde{\lambda}_{4}-\lambda_{4} \beta \tilde{\lambda}_{3} & -\lambda_{4} \alpha \tilde{\lambda}_{2}-\lambda_{3} \alpha \tilde{\lambda}_{1}+\lambda_{4} \beta \tilde{\lambda}_{1}-\lambda_{3} \beta \tilde{\lambda}_{2} \\
-\lambda_{2} \alpha \tilde{\lambda}_{4}-\lambda_{1} \alpha \tilde{\lambda}_{3}+\lambda_{2} \beta \tilde{\lambda}_{3}-\lambda_{1} \beta \tilde{\lambda}_{4} & \lambda_{2} \alpha \tilde{\lambda}_{2}+\lambda_{1} \alpha \tilde{\lambda}_{1}+\lambda_{1} \beta \tilde{\lambda}_{2}-\lambda_{2} \beta \tilde{\lambda}_{1}
\end{array}\right\|,
\end{gathered}
$$

where $\alpha(t)=\tilde{\alpha}(t)$ and $\beta(t)=-\tilde{\beta}(t)$ can be arbitrary symmetric and antisymmetric $N \times N$ matrices. Blocks $\lambda_{j}$ of a symplectic matrix $\Lambda$ satisfy many identities [6]:

$$
\begin{gathered}
\lambda_{1} \tilde{\lambda}_{2}=\lambda_{2} \tilde{\lambda}_{1}, \quad \lambda_{3} \tilde{\lambda}_{4}=\lambda_{4} \tilde{\lambda}_{3}, \quad \tilde{\lambda}_{1} \lambda_{3}=\tilde{\lambda}_{3} \lambda_{1}, \quad \tilde{\lambda}_{4} \lambda_{2}=\tilde{\lambda}_{2} \lambda_{4}, \\
\lambda_{4} \tilde{\lambda}_{1}-\lambda_{3} \tilde{\lambda}_{2}=\lambda_{1} \tilde{\lambda}_{4}-\lambda_{2} \tilde{\lambda}_{3}=E_{N}, \quad \tilde{\lambda}_{4} \lambda_{1}-\tilde{\lambda}_{2} \lambda_{3}=\tilde{\lambda}_{1} \lambda_{4}-\tilde{\lambda}_{3} \lambda_{2}=E_{N} .
\end{gathered}
$$

The case of $N=1$ is trivial and non-interesting because $\beta(t)=0$ and $B_{0} \sim E_{2}$. However, the situation can be non-trivial for $N \geq 2$. For example, we have the following set of possible matrices $\alpha(t)$ and $\beta(t)$ for $N=2$ :

$$
\alpha(t)=\left\|\begin{array}{ll}
r(t) & g(t) \\
g(t) & s(t)
\end{array}\right\|, \quad \beta(t)=\left\|\begin{array}{cc}
0 & f(t) \\
-f(t) & 0
\end{array}\right\| .
$$

Consequently, the invariant matrix $\mathcal{M}=\eta E_{2 N}$ exists for any time-dependent Hamiltonian of the form

$$
\hat{H}=\frac{1}{2}\left[r(t)\left(\hat{p}_{x}^{2}+\hat{x}^{2}\right)+s(t)\left(\hat{p}_{y}^{2}+\hat{y}^{2}\right)+2 g(t)\left(\hat{p}_{x} \hat{p}_{y}+\hat{x} \hat{y}\right)+2 f(t)\left(\hat{p}_{x} \hat{y}-\hat{x} \hat{p}_{y}\right)\right] .
$$

Introducing the standard bosonic annihilation and creation operators,

$$
\hat{a}=\frac{\hat{x}+i \hat{p}_{x}}{\sqrt{2}}, \quad \hat{a}^{\dagger}=\frac{\hat{x}-i \hat{p}_{x}}{\sqrt{2}}, \quad \hat{b}=\frac{\hat{y}+i \hat{p}_{y}}{\sqrt{2}}, \quad \hat{b}^{\dagger}=\frac{\hat{y}-i \hat{p}_{y}}{\sqrt{2}},
$$

one can re-write Hamiltonian (35) as

$$
\hat{H}=r(t)\left(\hat{a}^{\dagger} \hat{a}+1 / 2\right)+s(t)\left(\hat{b}^{\dagger} \hat{b}+1 / 2\right)+\xi(t) \hat{a} \hat{b}^{\dagger}+\xi^{*}(t) \hat{b} \hat{a}^{\dagger}, \quad \xi(t)=g(t)+i f(t) .
$$

This is a generalization of the frequency converter Hamiltonian considered in paper [1] with $r, s=$ const and $\xi(t)=\kappa e^{i \omega t}$. Further generalizations can be obtained by means of transformation (29). For example, using the simplest symplectic transformation with diagonal matrices $\lambda_{1}=\lambda_{4}^{-1}$ and $\lambda_{2}=\lambda_{3}=0$, one can introduce different masses and frequencies in the basic Hamiltonian (35). Then, the invariant matrix $\mathcal{M}$ will remain diagonal, but with different elements.

\section{Discussion}

The main result of this paper is the description of a general structure of the covariance matrices that do not depend on time for quadratic $N$-dimensional Hamiltonians. If the time independent Hamiltonian matrix $B$ (of dimension $2 N \times 2 N$ ) is positively definite, such invariant matrices depend on $2 N$ parameters for $N \geq 2$ (and a single parameter if $N=1$ ). If the symmetric matrix $B$ is only non-negatively definite, the situation depends on the degeneracy of zero eigenvalues of this matrix: the invariant matrices exist if the number of such eigenvalues is even (an example is a free charge moving in a homogeneous magnetic 
field as considered in Section 2); however, these do not exist if this number is odd (an example is the free particle Hamiltonian $\hat{H}=\hat{p}^{2} /(2 m)$ ).

A general structure of time-dependent quadratic Hamiltonians admitting invariant covariance matrices has been established. Such Hamiltonians are generalizations of the frequency converter Hamiltonians of quantum optics, considered in paper [1].

A few words regarding the place of the invariant states considered in this paper in the world of other quantum invariants appears relevant. If the quadratic Hamiltonian does not depend on time then there exist some specific linear combinations of a few second-order moments that do not depend on time: well known examples are the mean value of the energy (and angular momentum in the case of additional symmetries). Moreover, such kinds of specific linear combinations can be constructed for arbitrary time-dependent quadratic Hamiltonians, as was shown by Lewis and Riesenfeld [20]. Their method of time-dependent quantum operators and integrals of motion was generalized and further developed by Malkin and Man'ko with collaborators [6,21-31] and other authors [32-56].

The important applications of the Lewis-Riesenfeld and Malkin-Man'ko invariants include, e.g., the "inverse engineering" of quadratic Hamiltonians: a search of "shortcuts to adiabaticity" in different kinds of traps [57-60]. Other applications are related to the theory of the geometric (Berry) phase [61-67], invariants of non-Hermitian Hamiltonians [68-70], and open quantum systems [71-74].

Basic elements of the Malkin-Man'ko construction for Hamiltonian (1) are $2 \mathrm{~N}$ linear operator integrals of motion, combined in the vector $\hat{\mathbf{Q}}(t)=\Lambda(t) \hat{\mathbf{q}}$, where the $2 N \times 2 N$ symplectic matrix $\Lambda(t)$ satisfies the equation $d \Lambda / d t=\Lambda \Sigma B(t)$. Then, the mean value of any quadratic form $\hat{\mathbf{Q}}(t) G \hat{\mathbf{Q}}(t)=\hat{\mathbf{q}} \tilde{\Lambda}(t) G \Lambda(t) \hat{\mathbf{q}}$ with symmetric matrix $G$ does not depend on time. All such quadratic invariants are linear combinations of the second-order moments with time-dependent coefficients determined by the Hamiltonian matrix $B$.

In addition to these invariants (whose values depend on matrix $B$ and the initial quantum state), there exist other combinations of the second-order moments (not linear, but bilinear or multilinear) that depend on the initial state and the antisymmetric commutator matrix ( $\Sigma$ in the case of standard coordinates and momenta operators) but do not depend on the concrete matrix $B$. Such combinations were named universal quantum invariants in $[3,6,75,76]$. The simplest examples are "trace universal invariants" $\mathcal{L}_{2 m}=\operatorname{Tr}\left[\left(\mathcal{M} \Sigma^{-1}\right)^{2 m}\right]$ with $m=1,2, \ldots, N$. They obey the generalized uncertainty relations [77] $(-1)^{m} \mathcal{L}_{2 m} \geq N / 2^{2 m-1}$. Similar invariants and their special cases in the physics of particle and optical beams were considered, e.g., in the papers [78-92]. Such constructions are frequently used in quantum information theory under the name "symplectic invariants" [9,93,94].

The invariants mentioned above contain only some elements of the covariance matrix $\mathcal{M}$. Moreover, each of these elements can depend on time, and only their specific combinations are time-independent. On the contrary, all elements of the invariant matrices studied in this paper do not depend on time. This is a novelty suggested in [1]. What are invariant quantum states? For the Gaussian states, there exists the direct and unique relation between the covariance matrix and the state, given by Formula (11). In this case, the majority of invariant states is represented by quantum mixtures.

Pure invariant states are the vacuum (minimum energy) states (or coherent states, as soon as the displacement operator does not change the covariance matrix), although the situation may be more complicated for certain Hamiltonians (such as those including the magnetic field). A description of invariant non-Gaussian pure states could be an interesting avenue of future research. Another potentially interesting problem could be the search of invariant states for open quantum systems, when Equation (6) is replaced with a more general equation $d \mathcal{M} / d t=\mathcal{M} A+\tilde{A} \mathcal{M}+D$, where $D$ is a symmetric diffusion matrix and $A$ is a "drift" matrix containing the terms responsible for dissipation [3,95]. These subjects, however, require separate studies.

Funding: This research received no external funding. 
Acknowledgments: The author acknowledges the partial support of the Brazilian funding agency Conselho Nacional de Desenvolvimento Científico e Tecnológico (CNPq).

Data Availability Statement: Not applicable.

Conflicts of Interest: The author declares no conflict of interest.

\section{References}

1. López-Saldívar, J.A.; Man'ko, M.A.; Man'ko, V.I. Differential parametric formalism for the evolution of Gaussian states: Nonunitary evolution and invariant states. Entropy 2020, 22, 586. [CrossRef] [PubMed]

2. Robertson, H.P. An indeterminacy relation for several observables and its classical interpretation. Phys. Rev. 1934, 46, 794-801. [CrossRef]

3. Dodonov, V.V.; Man'ko, V.I. Density matrices and Wigner functions of quasiclassical quantum systems. In Group Theory, Gravitation and Elementary Particle Physics (Proceedings of Lebedev Physics Institute, Volume 167); Komar, A.A., Ed.; Nova Science: Commack, NY, USA, 1987; pp. 7-101.

4. Dodonov, V.V.; Horovits, M.B. Change of energy and magnetic moment of a quantum charged particle after a fast jump of the magnetic field in solenoids of arbitrary cross sections. Physica A 2021, 571, 125843. [CrossRef]

5. Agarwal, G.S. Wigner-function description of quantum noise in interferometers. J. Mod. Opt. 1987, 34, 909-921. [CrossRef]

6. Dodonov, V.V.; Man'ko, V.I. Evolution of multidimensional systems. Magnetic properties of ideal gases of charged particles. In Invariants and the Evolution of Nonstationary Quantum Systems (Proceedings of Lebedev Physics Institute, Volume 183); Markov, M.A., Ed.; Nova Science: Commack, NY, USA, 1989; pp. 263-414.

7. Braunstein, S.L.; van Loock, P. Quantum information with continuous variables. Rev. Mod. Phys. 2005, 77, 513-577. [CrossRef]

8. Akhundova, E.A.; Dodonov, V.V.; Man'ko, V.I. Wigner functions of quadratic systems. Phys. A 1982, 115, 215-231. [CrossRef]

9. Banchi, L.; Braunstein, S.L.; Pirandola, S. Quantum fidelity for arbitrary Gaussian states. Phys. Rev. Lett. 2015, 115, 260501. [CrossRef] [PubMed]

10. Gantmacher, F.R. Theory of Matrices; AMS Chelsea: Providence, RI, USA, 1959.

11. Johnson, M.H.; Lippmann, B.A. Motion in a constant magnetic field. Phys. Rev. 1949, 76, 828-832. [CrossRef]

12. Malkin, I.A.; Man'ko, V.I. Coherent states of a charged particle in a magnetic field. Zh. Eksp. Teor. Fiz. 1969, 55, 1014-1025; English translation: Sov. Phys.-JETP 1969, 28, 527-532.

13. Bogoliubov, N.N.; Tyablikov, S.V. An approximate method of finding the lowest energy levels of electrons in a metal. Zhurn. Eksp. Teor. Fiz. 1949, 19, 256-268. (In Russian)

14. Tyablikov, S.V. Methods in the Quantum Theory of Magnetism; Plenum: New York, NY, USA, 1967; pp. 105-113.

15. Bergman, E.E.; Holz, A. Exact solutions of an $n$-dimensional anisotropic oscillator in a uniform magnetic field. Nuovo Cim. B 1972, 7, 265-276. [CrossRef]

16. Titulaer, U.M. Ergodic features of harmonic-oscillator systems. I. Physica 1973, 70, 257-275. [CrossRef]

17. Tsallis, C. Diagonalization methods for the general bilinear Hamiltonian of an assembly of bosons. J. Math. Phys. 1978, 19, 277-286. [CrossRef]

18. Colpa, J.H.P. Diagonalization of quadratic boson Hamiltonians. Physica A 1978, 93, 327-343. [CrossRef]

19. Maldonaldo, O. On the Bogoliubov transformation for quadratic boson observables. J. Math. Phys. 1993, 34, 5016-5027. [CrossRef]

20. Lewis, H.R., Jr.; Riesenfeld, W.B. An exact quantum theory of the time-dependent harmonic oscillator and of a charged particle in a time-dependent electromagnetic field. J. Math. Phys. 1969, 10, 1458-1473. [CrossRef]

21. Malkin, I.A.; Man'ko, V.I.; Trifonov, D.A. Invariants and evolution of coherent states for charged particle in time-dependent magnetic field. Phys. Lett. A 1969, 30, 414-416. [CrossRef]

22. Malkin, I.A.; Man'ko, V.I.; Trifonov, D.A. Linear adiabatic invariants and coherent states. J. Math. Phys. 1973, 14, 576-582. [CrossRef]

23. Dodonov, V.V.; Malkin, I.A.; Man'ko, V.I. Integrals of the motion, Green functions and coherent states of dynamical systems. Int. J. Theor. Phys. 1975, 14, 37-54. [CrossRef]

24. Dodonov, V.V.; Malkin, I.A.; Man'ko, V.I. Invariants and the Green functions of a relativistic charged particle in electromagnetic fields. Lett. Nuovo Cim. 1975, 14, 241-244. [CrossRef]

25. Ivanova, E.V.; Malkin, I.A.; Man'ko, V.I. Invariants and radiation of some nonstationary systems. Int. J. Theor. Phys. 1977, 16, 503-515. [CrossRef]

26. Dodonov, V.V.; Man'ko, V.I. Coherent states and the resonance of a quantum damped oscillator. Phys. Rev. A 1979, 20, 550-560. [CrossRef]

27. Castaños, O.; López-Peña, R.; Man'ko, V.I. Noether's theorem and time-dependent quantum invariants. J. Phys. A Math. Gen. 1994, 21, 1751-1770. [CrossRef]

28. Man'ko, V.I.; Mendes, R.V. Time-dependent invariants for Dirac equation and Newton-Wigner position operator. Phys. Scr. 1997, 56, 417-422. [CrossRef]

29. Fedele, R.; Man'ko, V.I. Quantumlike corrections and semiclassical description of charged-particle beam transport. Phys. Rev. E 1998, 58, 992-1001. [CrossRef] 
30. Man'ko, V.I.; Rosa, L.; Vitale, P. Time-dependent invariants and Green functions in the probability representation of quantum mechanics. Phys. Rev. A 1998, 57, 3291-3303. [CrossRef]

31. Man'ko, V.I.; Markovich, L.A. Quantum tomography of time-dependent nonlinear hamiltonian systems. Rep. Math. Phys. 2019, 83, 87-106. [CrossRef]

32. Leach, P.G.L. Quadratic Hamiltonians, quadratic invariants and the symmetry group SU(n). J. Math. Phys. 1978, 19, 446-451. [CrossRef]

33. Dhara, A.K.; Lawande, S.V. Time-dependent invariants and the Feynman propagator. Phys. Rev. A 1984, 30, 560-567. [CrossRef]

34. Abe, S. Invariants for time-dependent fermion systems. Phys. Lett. A 1993, 181, 359-365. [CrossRef]

35. Mizrahi, S.S.; Moussa, M.H.Y.; Baseia, B. The quadratic time-dependent Hamiltonian: Evolution operator, squeezing regions in phase space and trajectories. Int. J. Mod. Phys. B 1994, 8, 1563-1576. [CrossRef]

36. Andrews, M. Invariant operators for quadratic Hamiltonians. Am. J. Phys. 1999, 67, 336-343. [CrossRef]

37. Mostafazadeh, A. Supersymmetric dynamical invariants. J. Phys. A Math. Gen. 2001, 34, 4493-4505. [CrossRef]

38. Guasti, M.F.; Moya-Cessa, H. Amplitude and phase representation of quantum invariants for the time-dependent harmonic oscillator. Phys. Rev. A 2003, 67, 063803. [CrossRef]

39. Abdalla, M.S.; Leach, P.G.L. Wigner functions for time-dependent coupled linear oscillators via linear and quadratic invariant processes. J. Phys. A Math. Gen. 2005, 38, 881-893. [CrossRef]

40. Choi, J.R.; Yeon, K.H. Quantum properties of light in linear media with time-dependent parameters by Lewis-Riesenfeld invariant operator method. Int. J. Mod. Phys. B 2005, 19, 2213-2224. [CrossRef]

41. Abdalla, M.S.; Choi, J.R. Propagator for the time-dependent charged oscillator via linear and quadratic invariants. Ann. Phys. 2007, 322, 2795-2810. [CrossRef]

42. Abdalla, M.S.; Leach, P.G.L. Lie algebraic treatment of the quadratic invariants for a quantum system. Theor. Math. Phys. 2009, 159, 535-550. [CrossRef]

43. Cherbal, O.; Drir, M.; Maamache, M.; Trifonov, D.A. Invariants and coherent states for a nonstationary fermionic forced oscillator. Phys. Lett. A 2010, 374, 535-538. [CrossRef]

44. Cordero-Soto, R.; Suazo, E.; Suslov, S.K. Quantum integrals of motion for variable quadratic Hamiltonians. Ann. Phys. 2010, 325, 1884-1912. [CrossRef]

45. Aldaya, V.; Cossío, F.; Guerrero, J.; López-Ruiz, F.F. The quantum Arnold transformation. J. Phys. A Math. Theor. 2011, 44, 065302. [CrossRef]

46. Fiore, G.; Gouba, L. Class of invariants for the two-dimensional time-dependent Landau problem and harmonic oscillator in a magnetic field. J. Math. Phys. 2011, 52, 103509. [CrossRef]

47. Bertin, M.C.; Pimentel, B.M.; Ramirez, J.A. Construction of time-dependent dynamical invariants: A new approach. J. Math. Phys. 2012, 53, 042104. [CrossRef]

48. Cruz, H.; Schuch, D.; Castaños, O.; Rosas-Ortiz, O. Time-evolution of quantum systems via a complex nonlinear Riccati equation. I. Conservative systems with time-independent Hamiltonian. Ann. Phys. 2015, 360, 44-60. [CrossRef]

49. Guerrero, J.; López-Ruiz, F.F. On the Lewis-Riesenfeld (Dodonov-Man'ko) invariant method. Phys. Scr. 2015, 90, 074046. [CrossRef]

50. Bagrov, V.G.; Gitman, D.M.; Pereira, A.S. Coherent states of systems with quadratic Hamiltonians. Braz. J. Phys. 2015, 45, 369-375. [CrossRef]

51. de Ponte, M.A.; Cônsoli, P.M.; Moussa, M.H.Y. Method for the construction of the Lewis-Riesenfeld time-dependent invariants and their eigenvalue equations. Phys. Rev. A 2018, 98, 032102. [CrossRef]

52. Lawson, L.M.; Avossevou, G.Y.H.; Gouba, L. Lewis-Riesenfeld quantization and $S U(1,1)$ coherent states for $2 \mathrm{D}$ damped harmonic oscillator. J. Math. Phys. 2018, 59, 112101. [CrossRef]

53. Zenad, M.; Ighezou, F.Z.; Cherbal, O.; Maamache, M. Ladder invariants and coherent states for time-dependent non-Hermitian Hamiltonians. Int. J. Theor. Phys. 2020, 59, 1214-1226. [CrossRef]

54. Zelaya, K.; Rosas-Ortiz, O. Quantum nonstationary oscillators: Invariants, dynamical algebras and coherent states via point transformations. Phys. Scr. 2020, 95, 064004. [CrossRef]

55. Zelaya, K.; Hussin, V. Time-dependent rational extensions of the parametric oscillator: Quantum invariants and the factorization method. J. Phys. A Math. Theor. 2020, 53, 165301. [CrossRef]

56. Zelaya, K.; Marquette, I.; Hussin, V. Fourth Painlevé and Ermakov equations: Quantum invariants and new exactly-solvable time-dependent Hamiltonians. J. Phys. A Math. Theor. 2021, 54, 015206. [CrossRef]

57. Chen, X.; Torrontegui, E.; Muga, J.G. Lewis-Riesenfeld invariants and transitionless quantum driving. Phys. Rev. A 2011, 83, 062116. [CrossRef]

58. Guéry-Odelin, D.; Ruschhaupt, A.; Kiely, A.; Torrontegui, E.; Martínez-Garaot, S.; Muga, J.G. Shortcuts to adiabaticity: Concepts, methods, and applications. Rev. Mod. Phys. 2019, 91, 045001. [CrossRef]

59. Muga, J.G.; Martínez-Garaot, S.; Pons, M.; Palmero, M.; Tobalina, A. Time-dependent harmonic potentials for momentum or position scaling. Phys. Rev. Res. 2020, 2, 043162. [CrossRef]

60. Tobalina, A.; Torrontegui, E.; Lizuain, I.; Palmero, M.; Muga, J.G. Invariant-based inverse engineering of time-dependent, coupled harmonic oscillators. Phys. Rev. A 2020, 102, 063112. [CrossRef] 
61. Morales, D.A. Correspondence between Berry's phase and Lewis's phase for quadratic Hamiltonians. J. Phys. A Math. Gen. 1988, 21, L889-L892. [CrossRef]

62. Mizrahi, S.S. The geometrical phase: An approach through the use of invariants. Phys. Lett. A 1989, 138, 465-468. [CrossRef]

63. Dodonov, V.V.; Man'ko, V.I. Adiabatic invariants, correlated states and Berry's phase. In Topological Phases in Quantum Theory (Proceedings of the International Seminar, Dubna, September 1988); Markovski, B., Vinitsky, S.I., Eds.; World Scientific: Bukit Batok, Singapore, 1989; pp. 74-83.

64. Gao, X.C.; Xu, J.B.; Qian, T.Z. Geometric phase and the generalized invariant formulation. Phys. Rev. A 1991, $44,7016-7021$. [CrossRef]

65. Mostafazadeh, A. Geometric phases, symmetries of dynamical invariants and exact solution of the Schrödinger equation. J. Phys. A Math. Gen. 2001, 34, 6325-6338. [CrossRef]

66. Mostafazadeh, A. Time-dependent Hilbert spaces, geometric phases, and general covariance in quantum mechanics. Phys. Lett. A 2004, 320, 375-382. [CrossRef]

67. Duzzioni, E.I.; Serra, R.M.; Moussa, M.H.Y. A general treatment of geometric phases and dynamical invariants. EPL 2008, 82, 20007. [CrossRef]

68. Gao, X.C.; Xu, J.B.; Qian, T.Z. Invariants and geometric phase for systems with non-hermitian time-dependent Hamiltonians. Phys. Rev. A 1992, 46, 3626-3630. [CrossRef] [PubMed]

69. Maamache, M.; Djeghiour, O.K.; Mana, N.; Koussa, W. Pseudo-invariants theory and real phases for systems with non-Hermitian time-dependent Hamiltonians. Eur. Phys. J. Plus 2017, 132, 383. [CrossRef]

70. Ramos, B.F.; Pedrosa, I.A.; de Lima, A.L. Lewis and Riesenfeld approach to time-dependent non-Hermitian Hamiltonians having $\mathcal{P} \mathcal{T}$ symmetry. Eur. Phys. J. Plus 2018, 133, 449. [CrossRef]

71. Dodonov, V.V.; Malkin, I.A.; Man'ko, V.I. Invariants and nonequilibrium density matrices. J. Stat. Phys. 1977, 16, 357-370. [CrossRef]

72. Dodonov, V.V.; Man'ko, V.I. Integrals of motion of pure and mixed quantum systems. Phys. A 1978, 94, 403-412. [CrossRef]

73. Kim, S.P.; Santana, A.E.; Khanna, F.C. Generalized invariants and quantum evolution of open fermionic systems. Phys. Lett. A 2000, 272, 46-52. [CrossRef]

74. Abe, S. Weak invariants of time-dependent quantum dissipative systems. Phys. Rev. A 2016, 94, 032116. [CrossRef]

75. Dodonov, V.V. Universal integrals of motion and universal invariants of quantum systems. J. Phys. A Math. Gen. 2000, 33, 7721-7738. [CrossRef]

76. Dodonov, V.V.; Man'ko, O.V. Universal invariants of quantum-mechanical and optical systems. J. Opt. Soc. Am. A 2000, 17, 2403-2410. [CrossRef]

77. Sudarshan, E.C.G.; Chiu, C.B.; Bhamathi, G. Generalized uncertainty relations and characteristic invariants for the multimode states. Phys. Rev. A 1995, 52, 43-54. [CrossRef]

78. Hernández, E.S.; Remaud, B. Quantal fluctuations and invariant operators for a general time-dependent harmonic oscillator. Phys. Lett. A 1980, 75, 269-272. [CrossRef]

79. Turner, R.E.; Snider, R.F. A phase space moment method for classical and quantum dynamics. Can. J. Phys. 1981, 59, 457-470. [CrossRef]

80. Simon, R.; Mukunda, N.; Sudarshan, E.C.G. Partially coherent beams and a generalized ABCD-law. Opt. Commun. 1988, 65, 322-328. [CrossRef]

81. Holm, D.D.; Lysenko, W.P.; Scovel, J.C. Moment invariants for the Vlasov equation. J. Math. Phys. 1990, 31, 1610-1615. [CrossRef]

82. Bastiaans, M.J. Second-order moments of the Wigner distribution function in first-order optical systems. Optik 1991, 88, 163-168.

83. Serna, J.; Martínez-Herrero, R.; Mejías, P.M. Parametric characterization of general partially coherent beams propagating through ABCD optical systems. J. Opt. Soc. Am. A 1991, 8, 1094-1098. [CrossRef]

84. Dragt, A.J.; Neri, F.; Rangarajan, G. General moment invariants for linear Hamiltonian systems. Phys. Rev. A 1992, 45, $2572-2585$. [CrossRef] [PubMed]

85. Bastiaans, M.J. ABCD law for partially coherent Gaussian light, propagating through first-order optical systems. Opt. Quant. Electron. 1992, 24, S1011-S1019. [CrossRef]

86. Martínez-Herrero, R.; Mejías, P.M.; Sanchez, M.; Neira, J.L.H. 3rd-order and 4th-order parametric characterization of partially coherent beams propagating through $A B C D$ optical systems. Opt. Quant. Electron. 1992, 24, S1021-S1026. [CrossRef]

87. Onciul, D. Invariance properties of general astigmatic beams through first-order optical systems. J. Opt. Soc. Am. A 1993, 10, 295-298. [CrossRef]

88. Dragoman, D. Higher-order moments of the Wigner distribution function in first-order optical systems. J. Opt. Soc. Am. A 1994, 11, 2643-2646. [CrossRef]

89. Atakishiyev, N.M.; Chumakov, S.M.; Rivera, A.L.; Wolf, K.B. On the phase space description of quantum nonlinear dynamics. Phys. Lett. A 1996, 215, 128-134. [CrossRef]

90. Martínez-Herrero, R.; Mejías, P.M. On the fourth-order spatial characterization of laser beams: New invariant parameter through ABCD systems. Opt. Commun. 1997, 140, 57-60. [CrossRef]

91. Sarris, C.M.; Caram, F.; Proto, A.N. The uncertainty principle as invariant of motion for time-dependent Hamiltonians. Phys. Lett. A 2004, 324, 1-8. [CrossRef] 
92. Sarris, C.M.; Proto, A.N. Time-dependent invariants of motion for complete sets of non-commuting observables. Phys. A 2005, 348, 97-109. [CrossRef]

93. Simon, R.; Mukunda, N.; Dutta, B. Quantum-noise matrix for multimode systems: $U(n)$ invariance, squeezing, and normal forms. Phys. Rev. A 1994, 49, 1567-1583. [CrossRef]

94. Serafini, A.; Illuminati, F.; De Siena, S. Symplectic invariants, entropic measures and correlations of Gaussian states. J. Phys. B At. Mol. Opt. Phys. 2004, 37, L21-L28. [CrossRef]

95. Dodonov, V.V.; Man'ko, O.V. Quantum damped oscillator in a magnetic field. Phys. A 1985, 130, 353-366. [CrossRef] 